\title{
Hadron Spectroscopy and $B$ Physics at RHIC
}

\author{
S. U. Chung, D. P. Weygand and H. J. Willutzki \\ Physics Department, Brookhaven National Laboratory, Upton, NY 11973. U.S.A.
}

\begin{abstract}
A description is given of the physics opportunities at RHIC regarding quark-gluon spectroscopy. The basic idea is to isolate with appropriate. triggers the sub-processes pomeron + pomeron $\rightarrow$ hadrons and $\gamma^{*}+\gamma^{*} \rightarrow$ hadrons with the aet effective mass of hadrons in the range (If 1.0 to 10.0 $\mathrm{GeV}$, in order to study the hadronic states composed of quarks and gluons. The double-pomeron interactions are expected to produce glueballs and hybrids preferentially, while the two-offshell-photon initial states should couple predominantly to quarkonia and multiquark states.

Of particular interest is the possibility of carrying out a $\mathrm{CP}$-riolation study in the self-tagging $B$ decays, $B_{d}^{0} \rightarrow K^{+} \pi^{-}$and $\bar{B}_{d}^{0} \rightarrow K^{-} \pi^{+}$.
\end{abstract}

This manuscript has been authored under contract number DE-AC02-76CH00016 with the U.S. Department of Energy. Accordingly, the U.S. Government retains a non-exclusive, rovaltv-free license to publish or reproduce the published form of this contribution, or allow others to do so, for U.S. Government purposes.

\section{DISCLAIMER}

This report was prepared as a: account of work sponsored by an agency of the United States Government. Neither the United States Government ror any agency thereof, nor any of their employees, makes any warranty, express or implied, or assumes any legal liability or responsibility for the accuracy, completeness, or usefulness of any information, apparatus, product, or process disclosed, or represents that its use would not infringe privately owned rights. Reference herein to any specific commercial product, process, or service by trade name, trademark,

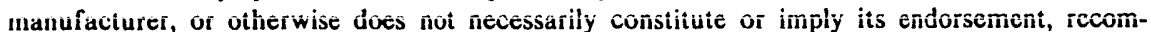
mendation, or favoring by the United States Government or any agency thereof. The views and opinions of authors expressed herein do not necessarily state or reflect those of the United States Government or any agency thereof. 


\section{Hadron Physics at RHIC}

In this section is described a conceptual design for carrying out a study of quark-gluon spectroscopy at the BNL Relativistic Heavy Ion Collider (RHIC) ${ }^{1}$. The current design is an updated, upgraded version of that presented at the Fourth Workshop ${ }^{2}$ on Experiments and Detectors for RHIC, held at BNL in July 1990.

The ideu is derived from a double-pomeron exchange trigger which was successfully implemented in $\mathrm{R} 807$ (an ISR experiment at CERN) ${ }^{3}$. The resulting $\pi^{+} \pi^{-}$(see Fig. 1) and $K^{+} K^{-}$spectra provided key ingredients in the study of $J^{P C}=0^{++}$states $^{4}$ with masses around $1.0 \mathrm{GeV}$.

For the trigger to succeed, it is necessary that for $p \times p$ the recoiling beam particles come off at a very small angle, $\theta \leq 2 \mathrm{mr}$. At $\mathrm{RHIC}$ energies this corresponds to installing a set of four 'Roman pots,' two on each side up and down, $10 \mathrm{~m}$ away from the intersection region. Precision $5 \times 5 \mathrm{~cm}$ mini-drift chambers and scintillation counters will be installed in each Roman pot to detect and trigger on the scattered beam particles. The intersection region will be instrumented with a $4 \pi$-detector consisting of cylindrical drift chambers, ring-imaging Cerenkov counters and lead-scintillator barrel counters, all within a $7.2 \mathrm{~m}$ long solenoid magnet with a $4.1 \mathrm{~m}$ coil diameter, patterned after the Mark $\mathrm{III}^{5}$ and the ARGUS apparatus.

It was shown in R807 that imposition of momentum balance in the direction perpendicular to that of the beam particles results in pure exclusive events, as follows:

$$
\begin{aligned}
& p p \rightarrow p\left(\pi^{+} \pi^{-}\right) p \\
& p p \rightarrow p\left(K^{+} K^{-}\right) p
\end{aligned}
$$

where the systems shown in parentheses indicate the particles detected in the central detector. In the proposed RHIC experiment, the central detector will be optinized for charged as well as neutral particles with momenta up to $3 \mathrm{GeV} / \mathrm{c}$, so that the following 

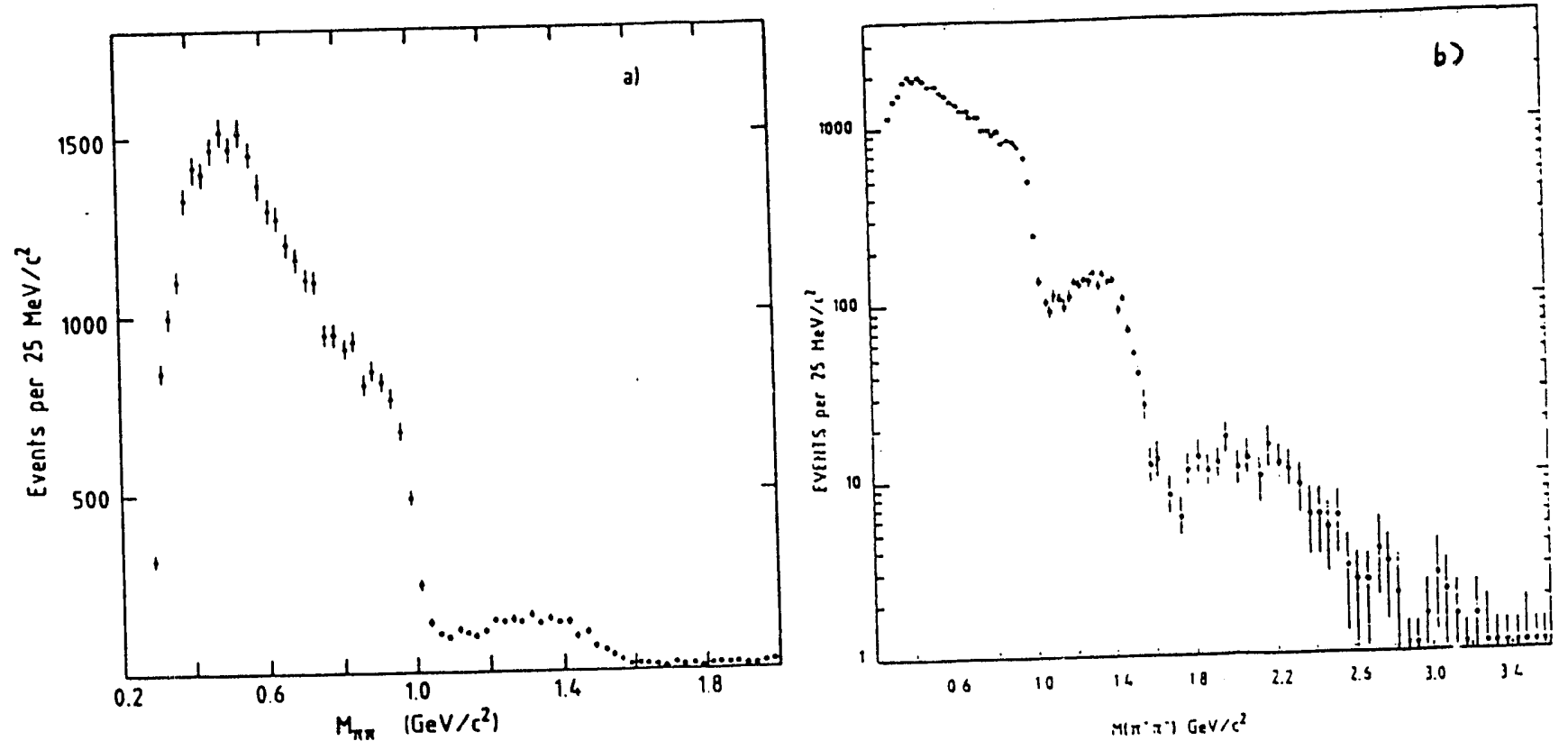

Figure 1: $\pi^{+} \pi^{-}$spectra for $p p \rightarrow p\left(\pi^{+} \pi^{-}\right) p$ at $\sqrt{s}=63 \mathrm{GeV}$. (a) Raw $M\left(\pi^{+} \pi^{-}\right)$spectrum. (b) Acceptance-corrected spectrum on log scale.

reactions can be studied:

$$
\begin{aligned}
& p p \rightarrow p(\eta \eta) p \\
& p p \rightarrow p(\omega \omega) p \\
& p p \rightarrow p(\phi \phi) p \\
& p p \rightarrow p(\eta \pi \pi) p \\
& p p \rightarrow p(\omega \pi \pi) p \\
& p p \rightarrow p(K \bar{K} \pi) p \\
& p p \rightarrow p(p \bar{p} \pi) p
\end{aligned}
$$

where the parentheses indicate again the central system. 
The momentum transfer squared from initial to final protons is given by

$$
-t \simeq(p \alpha)^{2} \simeq q^{2} \simeq 0.25(\mathrm{GeV} / \mathrm{c})^{2}
$$

where $p=250 \mathrm{GeV} / \mathrm{c}$ is the momentum of the initial proton and $\alpha \simeq 2 \mathrm{mr}$ is the scattering angle of the proton in the laboratory and $q \simeq 0.5 \mathrm{GeV} / \mathrm{c}$ is the momentum of the final proton perpendicular to the beam. Since the slope of $-t$ distributions is expected to be around $10 \mathrm{GeV}^{-2}$ at the top end of R.HIC energy ${ }^{6}$, the value $-t$ is sufficiently small to guarantee a pomeron exchange, and a double pomeron exchange reaction will result if both the final protons come off with $-t \leq 0.25(\mathrm{GeV} / \mathrm{c})^{2}$. In this case the central rapidity region corresponds in effect to the reaction

$$
\mathbf{P P} \rightarrow \text { hadrons }
$$

where $\mathbf{P}$ stands for a pomeron and the $\sqrt{s}$ for this subprocess ranges from 1.0 to $3.0 \mathrm{GeV}$. The upper limit on the $\sqrt{s}$ is not an inherent limitation; for a study of the states with $c(b)$ quarks, it should be extended to $5.0(10.0) \mathrm{GeV}$.

Let $M$ denote the invariant mass of the total hadronic system, i.e. the $\sqrt{s}$ for the process given above. Then,

$$
M^{2} \simeq \epsilon_{1} \epsilon_{2}(2 p)^{2}+t_{1}+t_{2}-2 \mathbf{q}_{1} \cdot \mathbf{q}_{2}
$$

where subscripts 1 and 2 denote final deflected beam particles and $1-\epsilon$ stands for the Feynman $x$ variables ${ }^{\top}$. Replacing $-t$ by $q^{2}$, one obtains

$$
M^{2} \simeq \epsilon_{1} \epsilon_{2}(2 p)^{2}-\left(q_{1}+q_{2}\right)^{2}
$$

From this one sees that

$$
\epsilon_{1} \sim \epsilon_{2} \sim \frac{M}{2 p} \simeq 4 \times 10^{-3}
$$

for $M=2 \mathrm{GeV}$ and $p=250 \mathrm{GeV} / \mathrm{c}$.

According to S. Y. Lee (BNL), one can choose an insertion mode in which the angular dispersion of the beam can be held to as low as $1.0 \mathrm{mr}$ at $10 \mathrm{~m}$ from the intersection. At this point, the deflected particles may range from $10 \mathrm{~mm}$ to $40 \mathrm{~mm}$ measured from the beam center. This corresponds to $q$ in the range of $0.25 \mathrm{GeV} / \mathrm{c}$ to $1.0 \mathrm{GeV} / \mathrm{c}$ for a proton 
beam at $250 \mathrm{GeV} / \mathrm{c}$. Within the Roman pots there will be a set of four drift-chamber modules and two scintillation counters, each with an active area measuring $50 \times 50 \mathrm{~mm}$.

The same experimental setup can be applied to heavy-ion collisions, e.g. those involving gold. M. Rhodes-Brown (BNL) points out that in the extreme low-momentum-transfer region the photon-photon interactions become competitive with the duuble-pomeron production,

$$
\sigma \sim(Z \alpha)^{4} \sim 0.1
$$

for $A u \times A u$ at $100 \mathrm{GeV} / \mathrm{u}$. The heavy ions of RHIC thus provide an opportunity for a study of two offshell-photon interactions,

$$
\gamma^{*} \gamma^{*} \rightarrow \text { hadrons }
$$

where $\sqrt{s}$ for this subprocess is in the range 1.0-3.0 GeV. Note that the photons involved can be highly offshell indeed; the $-t$ corresponding to the photon is given by

$$
-t \simeq q^{2}<(p \times 1 \mathrm{mr})^{2} \simeq 400(\mathrm{GeV} / \mathrm{c})^{2}
$$

where $p=197 \times 100 \mathrm{GeV} / \mathrm{c}$ and $1 \mathrm{mr}$ is the allowed angular dispersion of the beam.

The coherent production of hadrons by the two-photon process involves extremely sharp $-t$ distributions. According to A. Skuja and D. H. White ${ }^{8}$, the slope of the $-t$ distributions is $700 \mathrm{GeV}^{-2}$ for $A u \times A u$ at $100 \mathrm{GeV} / \mathrm{u}$, indicating that the beams simply pass through undeflected in the region where the cross section is appreciable. The energy loss is also extremely small,

$$
\epsilon_{1} \sim \epsilon_{2} \sim \frac{M}{2 p} \simeq 5 \times 10^{-5}
$$

for $M=2 \mathrm{GeV}$ and $p=197 \times 100 \mathrm{GeV} / \mathrm{c}$. It is seen that this loss factor is well within the allowed beam dispersion of RHIC.

It therefore follows that a proper $\gamma^{*} \gamma^{*}$ trigger calls for something other than the Roman pots, i.e. it has to rely on a veto on the deflected beam, by a set of four lead-scintillation sandwich barrel counters located at $10 \mathrm{~m}$ and $40 \mathrm{~m}$ away from the intersection point. A barrel counter consists of six truncated wedge detectors with width $5 \mathrm{~cm}$ and $20 \mathrm{~cm}$ and $50 \mathrm{~cm}$ long. Its design is identical to that of the EM calorineter in the central detector, as described in the section 3. Note that each barrel counter covers radial distances down to 


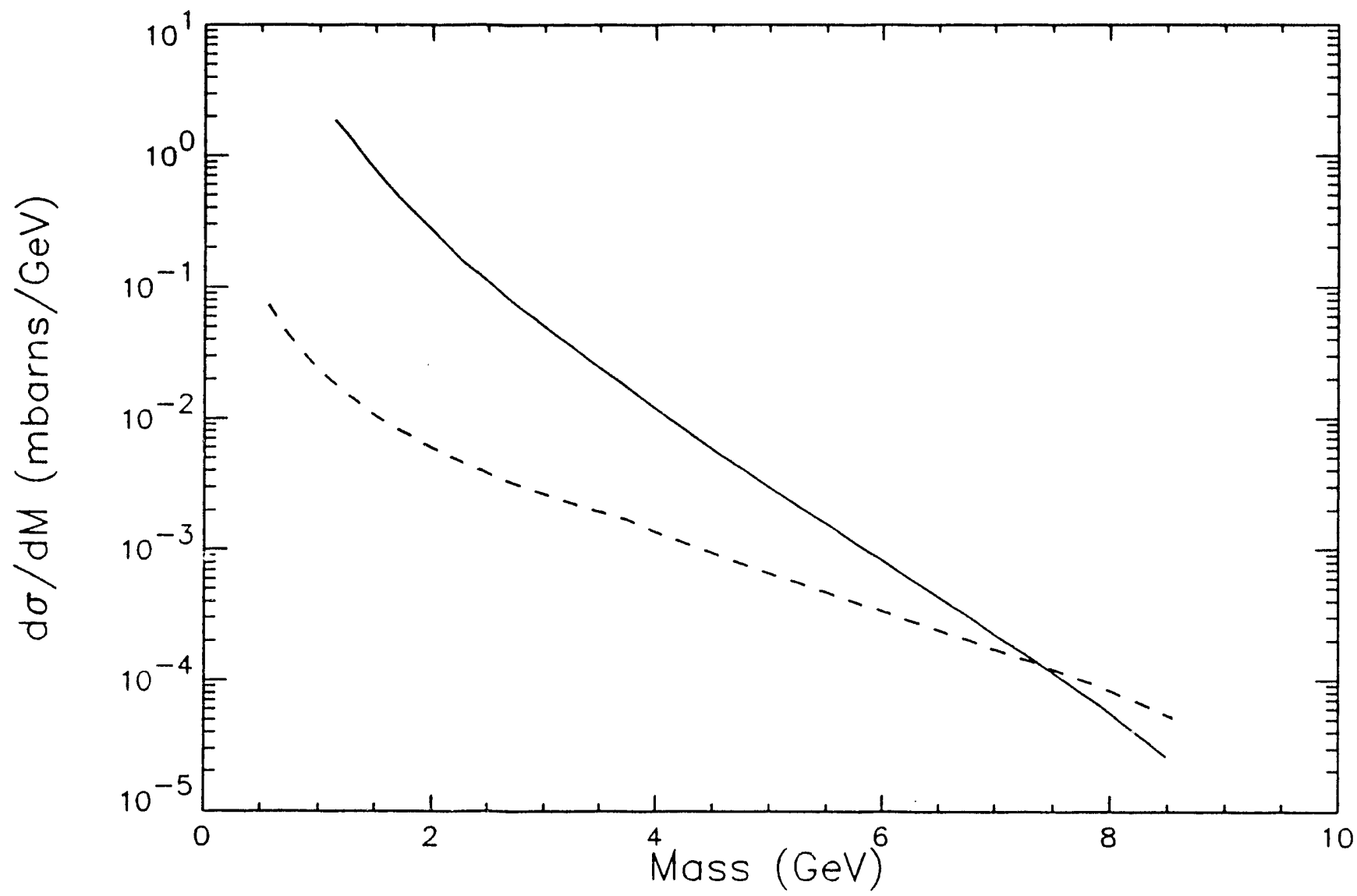

Figure 2: The cross section for two-photon processes as a function of mass $M$ of the central system with its rapidity limited to $|y|<1$ (the solid curve). The dashed curve corresponds to the same for double-Pomeron processes.

$5 \mathrm{~cm}$ radius from the beam line. With this setup, one can span the deflection angles from $1.25 \mathrm{mr}$ to $5 \mathrm{mr}$.

It is necessary, in addition, to veto on the diffractive dissociation of the beam. For this purpose, the end iron-plates of the magnet will be cut out at $100 \mathrm{~cm}$ radius, and a hadron calorimeter will be installed, which consists of 30 iron-scintillation sandwiches, designed to veto hadrons above $10 \mathrm{GeV} / \mathrm{c}$. $\therefore$ Aditional material on the calorimeter is given in the section 3 . 
The cross sections for production of a central system of mass $M$ through doublePomeron and two-photon processes have been calculated. ${ }^{9}$ Limiting the rapidity of the central system to $|y|<1$ and the mass $M$ between 1 and $10 \mathrm{GeV}$, one obtains $\sigma(\mathbf{P P}) \simeq$ $109 \mu \mathrm{b}$ for $p \times p$ at $100 \mathrm{GeV} / \mathrm{c}$ and $\sigma(\gamma \gamma) \simeq 1.11 \mathrm{mb}$ for $A u \times A u$ at $100 \mathrm{GeV} / \mathrm{u}$. The mass dependence of the cross sections is given in Fig. 2. The double-Pomeron cross section has roughly a $M^{-2}$ dependence, whereas the two-phoion cross section drops off much more sharply, even though it is some ten times larger than that of double-Pomeron process for small values of $M(M<3 \mathrm{GeV})$. This shows that two-photon processes are useful mainly for study of hadrons involving light-mass quarks.

What quantum numbers are allowed for the initial state? A Pomeron is not a physical particle, and it should behave as if a continuum of spin states is associated with the exchanged Regge trajectory. According to P. V. Landshoff (private communication), the first physically allowed state on the Pomeron trajectory is the $J^{P C}=2^{++}$state which may be the tensor glueball. Assuming a system of two Pomerons to be that of two $J^{P C}=$ $2^{++}$particles obeying the Bose-Einstein statistics, one can expect the system (with $I^{G}=$ $0^{+}$) to couple to all possible $J^{P}$ states with the constraint that $C$ and $L+S$ be even, where $L$ is the orbital angular momentum between the two Pomerons and $S$ is their total intrinsic spin. For the two-photon initial state, one may expect $I^{G}=0^{+}, 1^{-}$and $J^{P C}=(0,1,2,3,4, . .)^{++},(0,1,2,3,4, . .)^{-+}$. It should be noted that $J^{P C}=(1,3,5, . .)^{-+}$, allowed for both double-Pomeron and two-photon initial states, is exotic and cannot couple to quarkrnia.

It should be borne in mind that, given the sharp $-t$ distribution in heavy-ion interactions, the photons may in fact behave as if they were on-shell, i.e. zero-mass, especially for hadron effective masses greater than $1.5 \mathrm{GeV}$. In this case, the allowed values of $J^{P C}$ are more restricted; in particular, the spin-1 states do not couple to two real photons. It may be useful to work this result out in some detail. Consider a decay of a state of spin $J$ and parity $\eta$ into two identical particles of spin-1 with helicities $\lambda_{1}$ and $\lambda_{2}$; its decay auplitude is given by ${ }^{10,11}$

$$
A \propto F_{\lambda_{1} \lambda_{2}}^{J}\left[D_{M \lambda}^{J}(\phi, \theta, 0)\right]^{*}
$$


where the $D^{J}$ denotes the usual rotation function and $\lambda=\lambda_{1}-\lambda_{2}$. The spherical angles $(\theta, \phi)$ describe the orientation of the decay axis in the rest frame. The helicity decay amplitudes $F^{J}$ have the relationships, from parity conservation,

$$
F_{\lambda_{1} \lambda_{2}}^{J}=\eta(-1)^{J} F_{-\lambda_{1}-\lambda_{2}}^{J}
$$

and, from the fact that the decay particles are identical,

$$
F_{\lambda_{1} \lambda_{2}}^{J}=(-1)^{J} F_{\lambda_{2} \lambda_{1}}^{J}
$$

Note that, if $J=1, F^{J}=0$ if $\lambda_{1}=+1$ and $\lambda_{2}=-1$ since this would violate conservation of the z-component of spin along the decay axis. If the spin-1 decay particles are massless, then the zero helicities are not allowed. It is now easy to show that the allowed quantum numbers are

$$
J^{P C}=0^{ \pm+}, 2^{ \pm+}, 3^{++}, 4^{ \pm+}, 5^{++}, \ldots
$$

The symmetry relationships given above for the helicity amplitudes apply equally well when the decay particles have spin zero; one finds immediately that in this case one must have $J$ and $\eta$ even.

The initial state of the double-pomeron production is in reality a flavorless and colorless gluonic bundle. It follows therefore that the final state should be rich in gluonic excitations, i.e. glueballs and hybrids. In contrast, two offshell photons couple preferentially to charged quarks, e.g. $u \bar{u}$ or $c \bar{c}$ if the energy is high enough, leading to the production of quarkonia and multiquark states. 


\section{2. $B$ Physics at RHIC}

One of the more fundamental questions confronting the Standard Model based on the group $S U(3) \otimes S U(2) \otimes U(1)$ is the problem of understanding the origin of the CP violation and of the existence of three generations of quarks and leptons. In a conventional parameterization of the Standard Model, the CP violation arises from a single non-zero phase in the Cabbibo-Maskawa-Kobayasi (CKM) matrix. The study of the CP violation therefore involves, among others, precision measurements of all the elements of the CKM matrix.

It is now an accepted norm that, in addition to the $K^{-0} \vec{K}^{0}$ system, the CP-violation effects should occur substantially in the $B$ decays. There is therefore a tremendous push to start $B$ physics at LEP and TEVATRON. In addition, asymmetric $e^{+} e^{-}$machines as $B$ factories have been proposed at $\mathrm{SLAC}^{12}, \mathrm{KEK}^{13}$, Cornell ${ }^{14}$ and $\mathrm{CERN}^{15}$. A proposal also exists to build a dedicated detector ${ }^{16}$ for $B$ physics at the SSC.

With several crucial additions and modifications to the QGS, one can contemplate carrying out $B$ physics at RHIC. A possibility of $B$ physics at RHIC has been previously investigated by $\mathrm{N}$. Lockyer et $a{ }^{17}{ }^{17}$. In terms of certain key parameters, the QGS described above is essentially similar to the detector studied by them.

Of particular interest are the self-tagging $B$ decays: $B_{d}^{0} \rightarrow K^{+} \pi^{-}$and $\bar{B}_{d}^{0} \rightarrow K^{-} \pi^{+}$. The $P_{t}$ of the $B$ mesons at RHIC are typically about $4.4 \mathrm{GeV} / \mathrm{c}$. About $90 \%$ of the decay tracks are contained within \pm 2 units of pseudo-rapidity, and the $p_{t}$ of the $K^{ \pm}$ranges from $0.7 \mathrm{GeV} / \mathrm{c}$ to $7.0 \mathrm{GeV} / \mathrm{c}$, in contrast to the typical $300-\mathrm{MeV} / \mathrm{c}$ track for the underlying events. As $c \tau=350 \mu \mathrm{m}$ for the $B$ mesons, it is recessary to measure vertices at an accuracy of $10 \mu \mathrm{m}$ in order to be efficient in picking out $B$ events from the background.

These considerations argue for a micro-vertex detector, e.g. the silicon drift chamber ${ }^{18}$ with a position resolution in both $\mathrm{x}$ and $\mathrm{y}$ directions at $10 \mu \mathrm{m}$. It is envisioned that a three-layer barrel vertex detector will be fully instrumented for triggers, with a fast online processor called on to determine in real time the production and decay vertices. A $B$-trigger may be that in which a decay vertex is separated by more than $30 \mu \mathrm{m}$ from the production vertex. More details are given in the next section. 
At the highest luminosity for $p \times p$ at RHIC, there may be one to two interactions per bunch crossing, with the time separation between bunches expected to be $225 \mathrm{~ns}$. The drift-chamber module, described in the next section, has an average drift space of $5 \mathrm{~mm}$ and the maximum drift time of 50 to $100 \mathrm{~ns}$ depending on the type of gas mixture used. As the interaction diamond length is $\pm 22 \mathrm{~cm}$, multiple events from on a single bunch crossing can be resolved offline in principle as distinct clusters separated along the beam line.

The RHIC machine may operate in a $p \times p$ mode with a luminosity of $10^{32} \mathrm{~cm}^{-2} \mathrm{sec}^{-1}$ at $250 \times 250 \mathrm{GeV}$. The estimated $B \bar{B}$ cross section at these energies is about $10 \mu \mathrm{b}$, and a run of $10^{7} \mathrm{sec}$ would produce roughly $10^{10} B \bar{B}$ pairs. Assume that the fraction of $B_{d}^{0} \bar{B}_{d}^{0}$ pair production is $20 \%$ of that of the $B$ as a whole; that the branching ratio for $B_{d}^{0} \rightarrow K^{+} \pi^{-}$is $10^{-5}$; that the overall trigger and other efficiencies add up to $1 \%$. Then from a sample of $10^{10} B \bar{B}$ events, a CP asymmetry of $20 \%$ could be observed at the level of $3 \sigma$ effect.

This exercise shows that the $B$ physics at RHIC can be competitive with other machines. Other $B$ decay modes such as $B_{d}^{0} \rightarrow J / \psi K_{S}$ may not be as competitive.

\section{Central Detector at RHIC}

The central detector consists of a neutral and charged particle detection device with a $4 \pi$ coverage, all housed in a moderate-size solenoid magnet with an inner radius of 206 $\mathrm{cm}$ and $590 \mathrm{~cm}$ long outside. The magnet uses $\mathrm{Al}$ coils inside the yoke producing a field strength of $0.5 \mathrm{~T}$. It is designed to identify up to a dozen particles with momenta in the range $0.05-2.50 \mathrm{GeV} / \mathrm{c}$, for a study of meson systems with mass $1.0-3.0 \mathrm{GeV}$. The central detector is thus given the name QGS, for Quark-Gluon Spectrometer (see Fig. 3).

The QGS consists of a micro-vertex detector (MVD) located within a beam pipe, a drift-chamber module (CDC) surrounding the beam pipe, followed by two ring-imaging Cerenkov counters (RICH1 and RICH2), a time-of-flight (TOF) hodoscope and a leadscintillation sandwich EM calorimeter, all within the magnet coil. Each end of the magnet is instrumented with a hadron calorimeter. These items are described briefly below. 


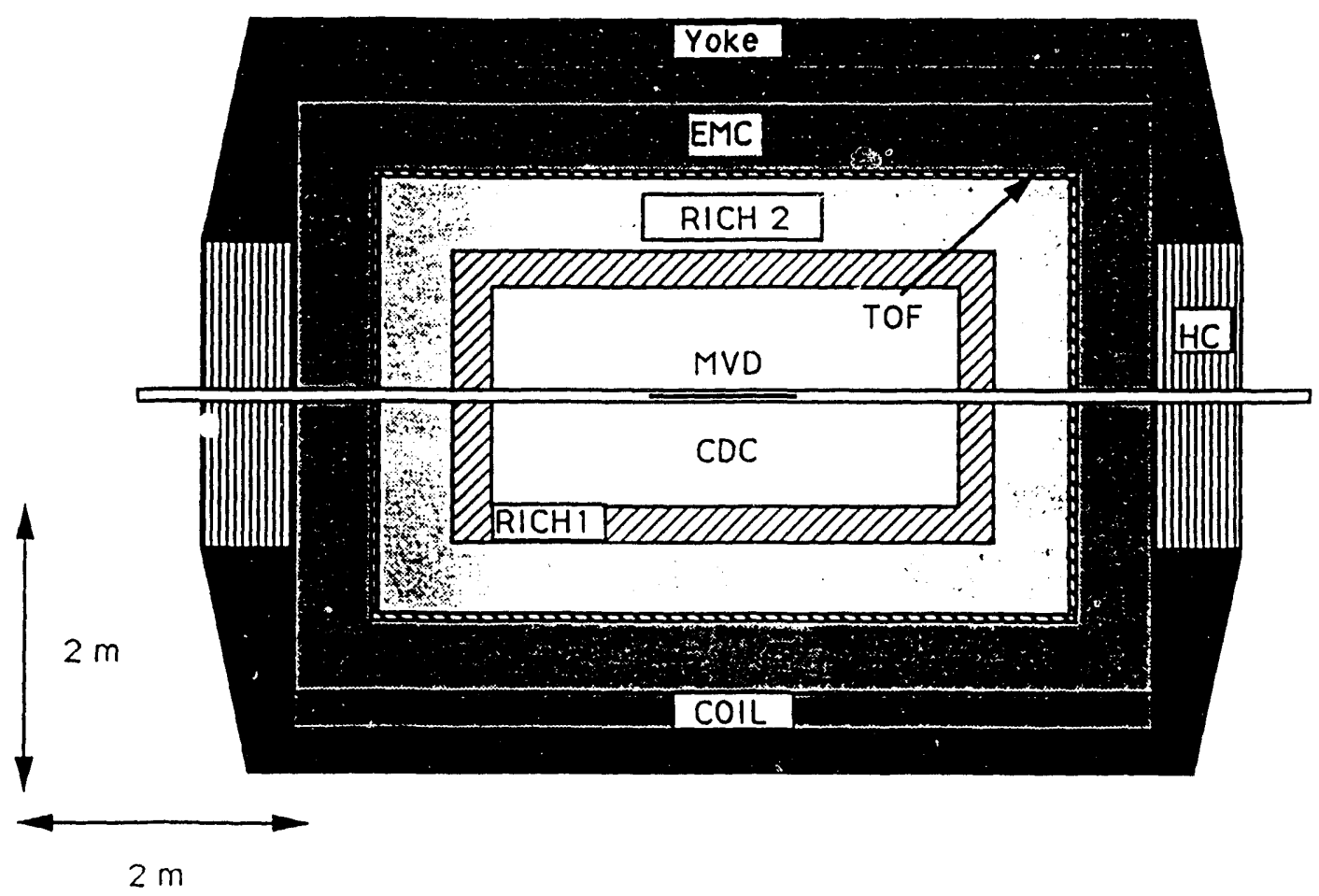

Figure 3: The Central detector: Quark-Gluon Spectrometer (QGS). The major components consist of a micro-vertex detector (MVD), a central drift-chamber module (CDC), two ring-imaging Cerenkov counters (RICH1 and RICH2), a time-of-flight hodoscope (TOF), an electromagnetic calorimeter (EMC), and a hadron calorimeter (HC).

The micro-vertex detector (MVD), shown in detail in Fig. 4, is envisioned to be a silicon drift chamber ${ }^{18}$ with its position resolution of $10 \mu \mathrm{m}$ in both $\mathrm{x}$ and $\mathrm{y}$ directions. The MVD is a three-layer barrel vertex detector, divided into four quadrants and fully instrumented for triggers. A fast on-line processor finds in real time the production and decay vertices in the $r-z$ plane, where the $z$ axis is along the beam line. An obvious advantage is that tracks are, to first order, linear in this plane, making the pattern recognition easier. Oftline analysis will involve finding tracks in the $r-\phi$ plane as well. A possible $B$ trigger is that 


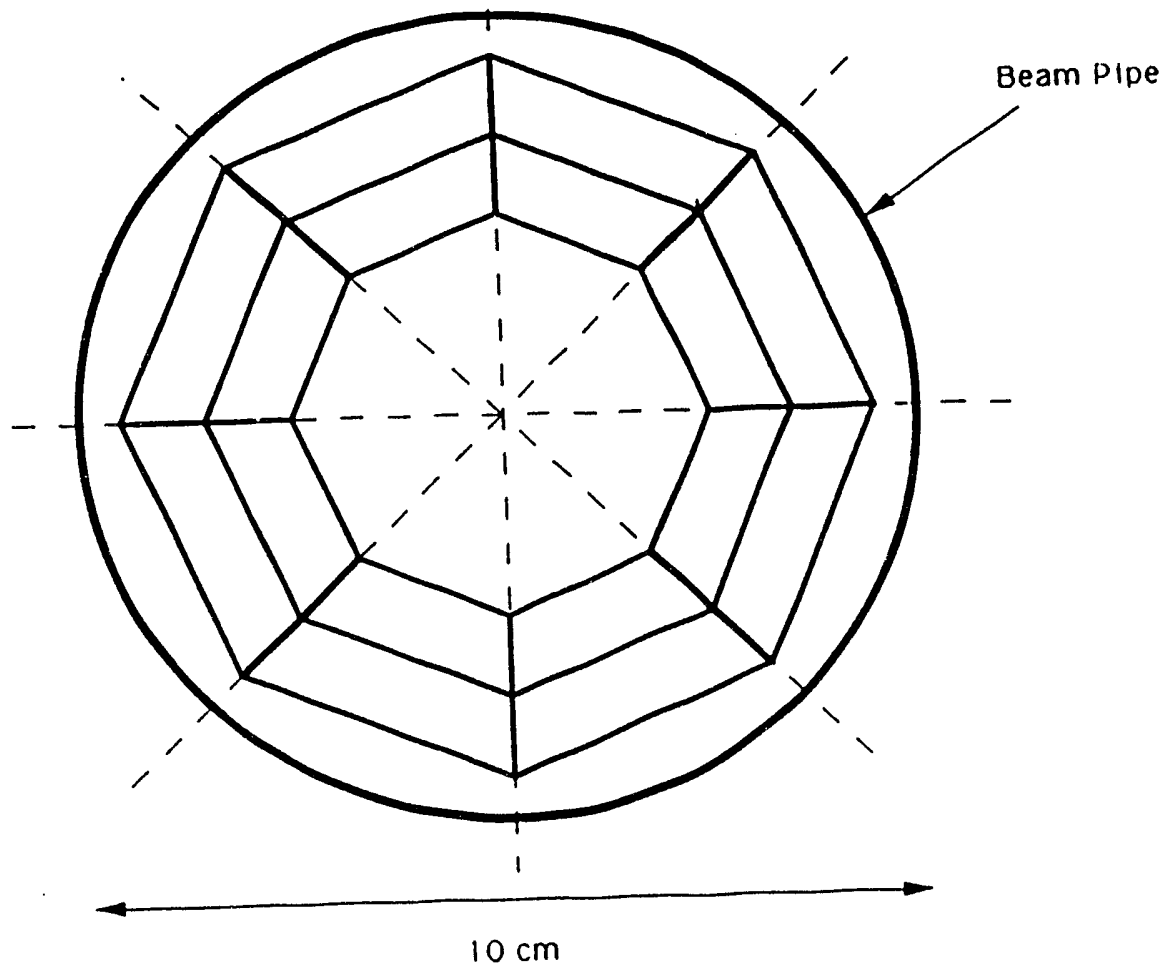

Figure 4: The micro-vertex detector (MVD) inside the beam pipe. It is in the shape of an octagon with four sectious, which can be retracted.

in which the decay vertex is separated by more than $30 \mu \mathrm{m}$ from the production vertex. This requires a very fast on-line computer or a dedicated micro-processor.

The beam-spot size for protons at $250 \mathrm{GeV} / \mathrm{c}$ can be approximated by Gaussian with $\sigma=0.6 \mathrm{~mm}$. Therefore, the vertex detector can be put as close as $5 \mathrm{~mm}$ from the beam center, when the RHIC is in a stable DC mode and the synchrotron radiation is properly shielded. However, for the time being, a more conservative design is adopted; the innermost layer of the MVD has an average radius of $2.4 \mathrm{~cm}$, and the second and third layer at radii of 3.3 and $4.2 \mathrm{~cm}$, respectively. The beam-pipe diameter for the QGS will be fixed at 10 cm. 
The drift-chamber module is $3.2 \mathrm{~m}$ long along the beam; it starts at a radius of $6 \mathrm{~cm}$ and extends to $76 \mathrm{~cm}$. The size of drift cells $(5 \mathrm{~mm})$ is dictated by the time interval of 225ns between bunch crossings. The whole module is divided into 9 layers, each containing two axial sense wires and two stereo wires at angles from $40 \mathrm{mr}$ to $80 \mathrm{mr}$. In all there will be some 9100 sense wires. The rms error on the transverse momentum is estimated to be

$$
\frac{\delta p_{\perp}}{p_{\perp}}=1.5 \% p_{\perp}(\mathrm{GeV} / \mathrm{c})
$$

assuming a measurement accuracy of $200 \mu \mathrm{m}$ and a field of $0.5 \mathrm{~T}$. The angular resolution is, from multiple scattering,

$$
\delta \theta=\frac{1.3 \mathrm{mr}}{p_{\perp}(\mathrm{GeV} / \mathrm{c})}
$$

Particle identification is provided by the $d E / d x$ measurement. Assuming an average of 36 measurements per track, the resolution is expected to be $15 \%$ FWHM. This provides a $3 \sigma$ $\pi / K$ separation up to about $0.6 \mathrm{GeV} / \mathrm{c}$.

At the highest luminosity for $p \times p$ at RHIC, there may be one to two interactions per bunch crossing, with the time separation between bunches expected to be $225 \mathrm{~ns}$. The drift-chamber module has an average drift space of $5 \mathrm{~mm}$ and the maximum drift time of 50 to $100 \mathrm{~ns}$ depending on the type of gas mixture used. As the interaction diamond length is $\pm 22 \mathrm{~cm}$, multiple events from on a single bunch crossing can be resolved offline in principle as distinct clusters separated alung the beam line. However, one may, for an extra margin of safety, decrease the drift space to $2.5 \mathrm{~mm}$, increasing the total number of sense wires to 18200 , and in addition use a gas mixture with a fast drift velocity of $100 \mu \mathrm{m} / \mathrm{ns}$, thus reducing the maximum drift time to $25 \mathrm{~ns}$.

In order to distinguish the mass of the $B_{d}^{0}$ from that of the $B_{s}^{0}$, a momentum resolution of the drift-chamber module better than that given above may be necessary; one may then have to replace the conventional solenoid magnet with a superconducting one and increase the field strength from $0.5 \mathrm{~T}$ to $2.0 \mathrm{~T}$, for a factor of four gain in the momentum resolution.

The RICH1 and RICH2 detectors envisaged here are patterned closely after the DELPHI design ${ }^{19}$. Several relevant formulae are first introduced here for convenience. Let $n$ be the index of refraction of a radiating mediun, and let $\beta$ be the velocity of a particle in 
the medium. Then, the threshold velocity or its $\eta=\beta \gamma$ and the Cerenkov angle are given by

$$
\begin{aligned}
\eta_{t} & =\frac{1}{\sqrt{n^{2}-1}}=\frac{1}{\sqrt{2 \delta+\delta^{2}}} \\
\tan \theta_{c} & =\sqrt{(n \beta)^{2}-1}=\beta \sqrt{\frac{1}{\eta_{t}^{2}}-\frac{1}{\eta^{2}}}
\end{aligned}
$$

where $\delta=n-1$. Let $L$ be the length of the radiator plus the drift space. Then, the radius of the Cerenkov ring is

$$
r=L \tan \theta_{c}=L \sqrt{(n \beta)^{2}-1}
$$

Or, expressing in terms of $\eta_{t}$, one obtains

$$
r=\left(\frac{L}{\eta_{t}}\right) \beta \sqrt{1-\left(\frac{\eta_{t}}{\eta}\right)^{2}}
$$

which shows that the maximum $r$ is given by $L / \eta_{t}$ for $p=\infty$. RICH1 extends from a radius of $76 \mathrm{~cm}$ to $101 \mathrm{~cm}$ and is $370 \mathrm{~cm}$ long on the outside. The front segment consists of a $1 \mathrm{~cm}$-thick liquid Freon $\left(C_{6} F_{14}\right)$ with an index of refraction $n=1.277\left(\eta_{t}=1.26\right)$, so that a relativistic particle produces Cerenkov light of $11.1 \mathrm{~cm}$ radius at the end of a 13 cm drift region $(L=14 \mathrm{~cm})$. The threshold momenta are 0.18 and $0.62 \mathrm{GeV} / \mathrm{c}$ for $\pi$ and $K$, respectively. It is then followed by a $4.4 \mathrm{~cm}$-thick photon-conversion region containing $\mathrm{CH}_{4}(75 \%)+\mathrm{C}_{2} \mathrm{H}_{6}(25 \%)$ and TMAE (Tetrakis Dimethyl Amino Ethylene). The readout is accomplished by a multi-wire proportional chamber with $1.5 \mathrm{~mm}$ wire spacing. The drift time is about $25 \mu \mathrm{s}$, which implies that this $\mathrm{RICH} 1$ counter is not a trigger device. The offline $\pi / K$ separation is impressive, starting at $0.18 \mathrm{GeV} / \mathrm{c}$ and extending to $3.0 \mathrm{GeV} / \mathrm{c}$. The upper limit is determined by the difference in radii of the $\pi$ and the $K$ rings, 11.1 and $10.7 \mathrm{~cm}$, respectively at $p=3.0 \mathrm{GeV} / \mathrm{c}$, and the experimental resolution of the rings as given by the wire spacing.

As the $K^{ \pm}$momenta range up to $10.0 \mathrm{GeV} / \mathrm{c}$ and above for $B$ decays, a second counter RICH2 will be added to handle momenta between 3.0 to $10.0 \mathrm{GeV} / \mathrm{c}$. It extends from a radius of $101 \mathrm{~cm}$ to $151 \mathrm{~cm}$ and is $470 \mathrm{~cm}$ long outside. Following the DELPHI design, the Cerenkov light is reflected back via parabolic mirrors to the proportional chamber, to be read out. The radiating medium is $C_{4} H_{10}$ (isobutane) at atmospheric pressure and room temperature with $\eta_{t}=19.8$. Assuming a nominal $L$ value of $100 \mathrm{~cm}$, the maximum $r$ is 
seen to be $5.05 \mathrm{~cm}$. For momenta between 2.8 and $9.8 \mathrm{GeV} / \mathrm{c}$, the RICH2 detector works as a threshold counter. Above 9.8 to $25 \mathrm{GeV} / \mathrm{c}$ the detector can separate a $K$ from a $\pi$; their Cerenkov rings at $p=25 \mathrm{GeV} / \mathrm{c}$ have radii of 5.0 and $4.6 \mathrm{~cm}$, respectively.

The TOF system is located at a radius of $151 \mathrm{~cm}$ and is $470 \mathrm{~cm}$ long. It consists of $1905 \times 5 \mathrm{~cm}$ scintillation counters, each viewed by two photomultipliers. The $\pi / K$ time separation for a path length $L$ is

$$
\Delta t(\mathrm{ps})=\left(\frac{100}{3}\right) L(\mathrm{~cm})\left(\frac{E_{K}}{p}-\frac{E_{\pi}}{p}\right)
$$

The resolution is conservatively estimated to be $250 \mathrm{ps}$, providing a $3 \sigma \pi / K$ separation from $0.08 \mathrm{GeV} / \mathrm{c}$ to $0.6 \mathrm{GeV} / \mathrm{c}$. Thus it can be used as an independent check of both the drift-chamber module and the RICH counters. It can also be used as a component in the charged particle triggers.

The EM calorimeter covers radii from $156 \mathrm{~cm}$ to $206 \mathrm{~cm}$ and is $580 \mathrm{~cm}$ long outside. In order to achieve good efficiency for soft photons, the detector is placed inside the magnet coil. It consists of $470010 \times 10 \mathrm{~cm}$ towers, each with 84 layers of $6 \mathrm{~mm}$ lead-scintillation sandwiches ( $1 \mathrm{~mm}$ of lead and $5 \mathrm{~mm}$ of plastic scintillator) for a total of $15 X_{0}$ and viewed by a photodiode through a wave-length shifter. The endcap EM calorimeters are similar in design, consisting of $153010 \times 10 \mathrm{~cm}$ towers. A similar device ${ }^{20}$ was used by ARGUS. The energy resolution is expected to be

$$
\frac{\delta E}{E}=\frac{7 \%}{\sqrt{E(\mathrm{GeV})}}
$$

for photon energies from $0.07 \mathrm{GeV}$ to $3.0 \mathrm{GeV}$.

The end caps of the magnet have cutouts with radius $100 \mathrm{~cm}$, and two hadron calorimeters with the active areas at radii from $5 \mathrm{~cm}$ to $100 \mathrm{~cm}$ will be installed in this space. Each calorimeter consists of 30 iron-scintillation disk-shape sandwiches. Both the iron plate and the plastic scintillator are $1 \mathrm{~cm}$ thick, and the periphery of the scintillator is edged with a wave-length shifter, which is read out by a photomultiplier. It is estimated that the energy resolution is

$$
\frac{\delta E}{E}=\frac{60 \%}{\sqrt{E(\mathrm{GeV})}}
$$


so that a $10 \mathrm{GeV} / \mathrm{c}$ particle can be measured with an accuracy of about $20 \%$. For the $A u \times A u$ run, the two hadron calorimeters will be used to veto on any particle with energy greater than $10 \mathrm{GeV} / \mathrm{c}$. It is expected that about $90 \%$ of all the diffractive dissociation events can thus be eliminated at the trigger level.

\section{Triggers at RHIC}

The trigger for PP interactions relies on a set of four scintillation counters within the Roman pots. For $p \times p$ runs, two triggers are possible with the Roman pots, 'up-up' and 'down-down.' This means that both of the counters above (below) the beam line at either side of the intersection region are triggered for 'up-up' ('down-down'). The triggers will be augmented with signals from the QGS, utilizing among others the hits in the EM calorimeter. Each hit above the minimum energy threshold, but below the maximum allowed energy, e.g. $10 \mathrm{GeV}$, is treated with equal weight; a fast microprocessor sums up independently the $x, y$ and $z$ projections of the location of the hit with respect to the midpoint of the intersection region. The trigger requires that the three sums are within a small preset range. This algorithm ensures that an event with a large missing energy in any direction will be eliminated, on the average. Note also that this technique treats charged and neutral particles on an equal footing.

For $A u \times A u$ runs, instead of the Roman pots, the trigger relies primarily on signals from the QGS to pick out two-photon events, accompanied by vetos at two end-cap hadron calorimeters and the four lead-scintillation barrel counters located $10 \mathrm{~m}$ and $40 \mathrm{~m}$ away from the intersection region. The vetoes guard against the small-angle beam deflections and the diffractive dissociation of the beams.

A Monte Carlo study is planned to assess the efficacy of the $x-, y$ - and z-projection methods described above in selecting production of low-mass hadrons in the central region.

The key to a successful study of the $B$ decays, $B_{d}^{0} \rightarrow K^{+} \pi^{-}$and $\bar{B}_{d}^{0} \rightarrow K^{-} \pi^{+}$, at RHIC lies in detecting a decay vertex, a $V$, separated from the production vertex at the trigger level. Given $c \tau=350 \mu \mathrm{m}$ for the $B$ and a typical $B$ momentum around $5 \mathrm{GeV} / \mathrm{c}$, it is desirable to be able to detect a $V$ separated by $50 \mu \mathrm{m}$ or less from the production vertex. A fast online computer or a dedicated micro-processor will be used to find in the 
$r-z$ plane the production vertex first, and then a $V$ separated from the production vertex by at least $30 \mu \mathrm{m}$. It is possible that the same algorithm can be applied to the $r-\phi$ plane as well, for detection at trigger level of a clean $V$. One may also rely on detection of a charged $K$ for a higher level trigger; however, a more careful Mote Carlo study will be needed for this and other possible trigger schemes.

\section{Conclusions}

In this note a brief description is given of an exciting opportunity to carry out hadron spectroscopy experiments at RHIC. The machine is slated to be ready for pliysics in 1997 . As such, it provides timely opportunities for further investigation into the outstanding problems in hadron physics, in particular those that cannot easily be tackled with the machines currently available in the world.

The key idea for RHIC is that by concentrating on the extreme double-peripheral region, the machine is used to produce hadronic systems at low $\sqrt{s}$ in the range $1.0-10.0 \mathrm{GeV}$.

The sub-processes responsible for the hadronic system in the central region may be expressed either as pomeron + pomeron $\rightarrow$ hadrons or as $\gamma^{*}+\gamma^{*} \rightarrow$ hadrons. The doublepomeron interactions are expected to produce glueballs and hybrids preferentially, while the two-offshell-photon initial states should couple predominantly to quarkonia and multiquark states. A whole gamut of $J^{P C}$-exotic mesons $\left(0^{+-}, 0^{--}, 1^{-+}, 2^{+-}, 3^{-+}, 4^{+-}, ..\right)$may be seen either directly in both types of interactions or in association with a single recoil photon in the final state. Another important distinction is that the hadronic system from a double-pomeron interaction has zero net flavor, whereas an $I^{G}=1^{-}$meson can couple readily to a two-photon initial state. The salient feature of this proposal lies in the fact that, for the first time, a study of the pomeron-pomeron interactions can be mounted with the same experimental setup as that of the photon-photon interactions.

In addition, the QGS with a micro-vertex detector and two RICH counters can tackle the CP-violation effects in the $B$ decays. The key element in this effort would have to be the capability to trigger on the $B$-decay vertices separated by more than $30 \mu \mathrm{m}$ from the 
production vertex. The upgraded QGS may also serve as the apparatus for a study of $\chi_{c}$ and $\chi_{b}$ states.

\section{Acknowledgements}

The authors are indebted to P. Rehak, S. Protopopescu and F. Paige for several helpful discussions.

\section{DISCLAIM QR}

This report was prepared as an account of work sponsored by an agency of the United States Government. Neither the United States Government nor any agency thereof, nor any of their employees, makes any warranty, express or implied, or assumes any legal liability or responsibility for the accuracy, completeness, or usefulness of any information, apparatus, product, or process disclosed, or represents that its use would not infringe privately owned rights. Reference herein to any specific commercial product, process, or service by trade name, trademark, manufacturer, or otherwise does not necessarily constitute or imply its endorsement, recommendation, or favoring by the United States Government or any agency thereof. The views and opinions of authors expressed herein do not necessarily state or reflect those of the United States Government or any agency thereof. 


\section{References}

1. Conceptual Design of the Relativistic Heavy Ion Collider (RHIC), BNL, Upton, NY (May 1989), BNL-52195.

2. S. U. Chung, W. Kern and H. J. Willutzki, 'Hadron Spectroscopy at RHIC,' Proc. Fourth Workshop on Experiments and Detectors at RHIC, BNI, July 1990, BNL52262 (1990), p. 207.

3. T. Akesson et al., Phys. Lett. 133B, 268 (1983);

T. Akesson et al., Nucl. Phys. B264, 154 (1986);

P. C. Cecil, Ph. D. Thesis, Cavendish Laboratory, Cambridge, England, Rutherford preprint RAL -T -004 (1984).

4. K. L. Au, D. Morgan and M. R. Pennington, preprint RAL -86 -076 (1986), Phys. Rev. D35, 1633 (1987).

5. D. Bernstein et al., NIM Vol. 226, 301 (1984).

6. K. Goulianos, 'Diffractive interactions of hadrons at high energies,' Physics Reports, Vol. 101, No. 3 (1983).

7. S. U. Chung, 'Kinematics, Phase Space and Phenomenological Amplitudes for DoublePomeron Exchange Reactions,' BNL Internal Report OG-734 (1983).

8. A. Skuja and D. H. White, 'Two-Photon Physics at RHIC,' RHIC Workshop, BNL51921 (1985), p. 289.

9. S. U. Chung, D. P. Weygand and H. J. Willutzki, 'Double-Pomeron and Two-Photon Processes at RHIC,' BNL-QGS-02-91.

10. M. Jacob and G. C. Wick, Annals of Physics Vol. 7, 404 (1959).

11. S. U. Chung, 'Spin Formalisms,' CERN Yellow Report, CERN 71-8 (1971).

12. The Physics Program of High-luminosity Asymmetric $B$ Factory at SLAC, SLAC-353 (October 1989). 
13. H. Aihara, $B$ Factory at KEK, Proceedings of the U.C.L.A. Workshop on Linear Collider $B \bar{B}$ Factory-Conceptual Design (January 1987), edited by D. Stork.

14. The Workshop towards establishing a b Factory, Syracuse Univ., Syracuse, NY (September 1989), edited by M. Goldberg and S. Stone.

15. Feasibilty Study for a $B$-meson Factory in the CERN ISR Tunnel, T. Nakada (Editor), CERN 90-02.

16. Expression of Interest for a Bottom Collider Detector at the SSC (May 1990), N. Lockyer (Spokesperson, Univ. of Pennsylvania).

17. Lockyer, et al., Proceedings of the Third Workshop on Experiments and Detectors for RHIC (July i990), p. 157.

18. P. Rehak et al., NIM Vol. A248, 367 (1986).

19. R. Arnold, et al., NIM Vol. 270, 255 (1988); NIM Vol. 270, 289 (1988).

20. W. Hofmann et al., NIM Vol. 163, 77 (1979). 


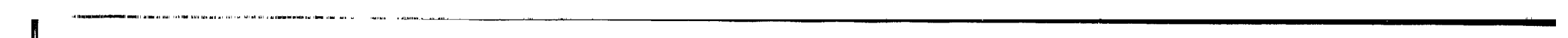

